\title{
Due lettere inedite di Gilles Ménage
}

\section{Vittorio Fortunati}

\section{(2) OpenEdition}

\section{Journals}

\section{Edizione digitale}

URL: https://journals.openedition.org/studifrancesi/40186

DOI: 10.4000/studifrancesi.40186

ISSN: 2421-5856

\section{Editore}

Rosenberg \& Sellier

\section{Edizione cartacea}

Data di pubblicazione: 1 juillet 2004

Paginazione: 129-132

ISSN: 0039-2944

\section{Notizia bibliografica digitale}

Vittorio Fortunati, «Due lettere inedite di Gilles Ménage», Studi Francesi [Online], 142 (XLVIII | I) | 2004, online dal 30 novembre 2015, consultato il 09 septembre 2021. URL: http://journals.openedition.org/ studifrancesi/40186 ; DOI: https://doi.org/10.4000/studifrancesi.40186

\section{(c) $(1)(9)$}

Studi Francesi è distribuita con Licenza Creative Commons Attribuzione - Non commerciale - Non opere derivate 4.0 Internazionale. 


\section{Due lettere inedite di Gilles Ménage}

I corrispondenti italiani di Gilles Ménage (1613-1692) gravitavano, per la maggior parte, intorno all'Accademia della Crusca, a cui anche il francese era affiliato ${ }^{1}$. Tra essi, oltre ai più noti Carlo Dati ${ }^{2}$ e Francesco Redi ${ }^{3}$, va incluso il prelato Ottavio Falconieri (1636-1675). Romano, ma di origine fiorentina, Falconieri ricopri diverse cariche ecclesiastiche, tra cui quella di 'consultore'della Congregazione dell'Indice (1666), di 'cameriere segreto'del papa (1670) e di internunzio apostolico nelle Fiandre (1672). I suoi interessi intellettuali si concentrarono soprattutto sugli studi archeologici: tra i suoi saggi, i più importanti sono il De Nummo Apamensi ${ }^{4}$ (Roma, 1667) e le Inscriptiones athleticae nuper repertae (Ivi, 1668). Falconieri non disdegnò tuttavia le scienze, in particolare la matematica e l'astronomia: anzi, fu uno dei primi sostenitori del metodo galileiano. Tra le sue opere letterarie, si possono citare l'ode Urania (1655), composta in occasione dell'ascesa al Soglio pontificio del papa Alessandro VII e il Plausus trilinguis (1656), scritto in italiano, latino e greco in onore della regina Cristina di Svezia, giunta a Roma dopo la conversione al cattolicesimo. Non vanno, infine, dimenticati gli interventi del prelato nei dibattiti culturali contemporanei, come, per esempio, la sua presa di posizione, nell'ambito dell'Accademia della Crusca, a difesa dell'opera di Torquato Tasso contro i suoi detrattori'.

La copia manoscritta di due lettere di Gilles Ménage a Ottavio Falconieri si trova in un codice che ha per titolo Epistolae Mss. virorum illustrium ad Octavium Falconerium Romanum Sanctae Congregationis Indicis consultorem E ad alios, a cura di Giulio Tomitano e Giusto Fontanino ${ }^{6}$, datato 1783 e conservato presso la Biblioteca Nazionale Marciana di Venezia ${ }^{7}$. In base al registro dei lettori annesso al codice, non risulta che i due testi siano mai stati copiati prima d'ora. Inoltre, lettere di Ménage a Falconieri non sono comprese nelle Mescolanze di Egidio Menagio ${ }^{8}$, che raccolgono diverse missive dell'autore francese a destinatari italiani.

La prima lettera riportata dal codice veneziano è datata 2 marzo 1669, mentre la seconda presenta una datazione incompleta: «À Paris ce mardy 26. Juin». Vi sono, tuttavia, elementi sufficienti per stabilire che la lettera venne scritta nel 1663. In primo luogo, Ménage fa riferimento alla partenza per la Normandia del marchese di Montausier, che proprio in quell'anno fu nominato governatore della provincia. Poi,

1) Cf. E. Samfiresco, Ménage. Polemiste, philologue, poète, Paris, 1902, Genève, Slatkine, 1971, pp. $24-25$.

(2) Quasi tutte le lettere di Ménage a C. Dati, conservate alla Biblioteca Nazionale Centrale di Firenze, sono state pubblicate da L. PéLISSIER in Documents annotés (Paris, 1891).

(3) E. SAMFIRESCO, op. cit., p. 24.

(4) Apamèa era una città della Frigia, fondata da
Antioco I di Siria

(5) E. Bonora, Torquato Tasso, in E. CECCHI - N. SAPEGNO (edd.), Storia della Letteratura Italiana, IV, Il Cinquecento, pp. 792-793.

(6) Il codice non precisa chi abbia materialmente copiato le lettere.

(7) Cod. Lat. 97 (= 4085).

(8) Paris, L. Bilaine, 1678. 
la lettera contiene un errata corrige, relativa a una poesia latina di Ménage, che si ritrova in una lettera a P.-D. Huet del 6 giugno 1663'. Infine, nell'anno 1663 il 26 giugno cadde, appunto, di martedì. Ci è sembrato, quindi, opportuno invertire l'ordine delle lettere nel codice, per ripristinare la corretta successione cronologica.

La corrispondenza tra Ménage e Falconieri doveva essere in corso fin dal 1660, come prova una lettera del francese a P.-D. Huet, scritta nel gennaio di quell'anno: «j'ay receu les six exemplaires de vostre Epistre [...] J'en ay envoyé un à Rome à Mr. Falconieri» ${ }^{10}$. Dal contenuto delle due lettere si può desumere, inoltre, che lo scambio epistolare fosse costante e piuttosto intenso ( $\ll$ je ne manqueray pas de vous les ${ }^{11}$ envoyer tout aussitôt»; «J'ay reçu les deux Lettres qu'il vous a plû m'escrire»; «Je vous envoyerai par la premiere occasion mes origines de la Langue Italienne»). Si tratta, come si può immaginare, della tipica corrispondenza fra due eruditi: lo scrivente parla di testi pubblicati in quegli anni e sollecita il giudizio del destinatario sulle proprie opere. Non mancano, però, allusioni ad avvenimenti d'attualità, quali il processo a Fouquet e le polemiche tra i gesuiti e i giansenisti.

La grafia del manoscritto è piuttosto chiara e coerente, il che ci ha permesso di trascrivere le lettere senza particolari difficoltà d'interpretazione. Naturalmente, non trattandosi di autografi, non vi è la certezza che l'ortografia di Ménage sia stata sempre rispettata. Abbiamo comunque riprodotto fedelmente i testi, limitandoci a intervenire in quei pochi casi in cui ci è parso di ravvisare un errore del copista. Per esempio, nella lettera del 26 giugno 1663 abbiamo corretto «je ne pensè» con «je ne pense». Inoltre abbiamo ripristinato l'iniziale maiuscola nei nomi «Ulysses» e «Valois».

Per concludere, desideriamo ringraziare la dottoressa Susy Marcon, conservatore dell'Ufficio Manoscritti della Biblioteca Nazionale Marciana, che ci ha assistiti nella nostra ricerca. Un sentito ringraziamento va, inoltre, alla professoressa Lea Caminiti Pennarola, dell'Università «Federico II» di Napoli, per le preziose informazioni forniteci riguardo alle edizioni a stampa delle lettere di Ménage.

VITTORIO FORTUNATI

A Paris ce mardy 26. Juin [1663] $]^{12}$

Je vous envoye le memoire des Livres de l'histoire Byzantine, qui ont été imprimés au Louvre ${ }^{13}$, avec le prix que les Libraires les veulent vendre. Quand vous l'aurés reçu, vous me ferés sçavoir s'il vous plaît si vous desirés, que je vous les achete, et je ne manqueray pas de vous les envoyer tout aussitôt. J'ay fait tenir vôtre Lettre à M.r de Valois ${ }^{14}$. S'il me donne son Ammian Marcellin pour vous, ce que je ne pense pas qu'il fasse, car il y a longtems que tous les Exemplaires en ont été distribués, je ne manqueray pas aussi de vous le faire tenir. Mon homme ${ }^{15}$ a oublié de corriger dans le volume de mes Poësies ${ }^{16}$ que je vous ay envoyé une faute d'edition, qui fait un sens tout contraire à ma pensée. C'est à la derniere page.

(9) G. Ménage, Lettres inédites à Pierre-Daniel Huet, ed. L. Caminiti Pennarola, Napoli, Liguori, 1993, pp. 187-188.

(10) Ivi, p. 89.

(11) Si riferisce ad alcune pubblicazioni storiche di cui Ménage parla nella lettera del 26 giugno 1663.

(12) Epistolae cit., p 258.

(13) A partire dalla metà del XVII secolo, presso la stamperia del Louvre venne pubblicato il corpus completo della storiografia bizantina (Cf. G.
Ostrogorsky, Storia dell'impero bizantino, trad. it. Torino, Einaudi, 1993, pp. 4-5).

(14) Si tratta con ogni probabilità di Henri de Valois (1603-1676), uno dei più importanti filologi dell'epoca, specialista dell'edizione di testi dell'Antichità.

(15) È il segretario Fleuri (cf. Lettres inédites à Pierre-Daniel Huet cit., p. 187).

(16) Un'edizione dei Poemata di Ménage venne pubblicata ad Amsterdam ne1 1663, presso la tipografia elzeviriana. 
Non culpandus erat, sed erat damnandus Ulysses. Il faut sed erat laudandus, Ulysses. Vous ferés s'il vous plaît, corriger cette faute dans vôtre exemplaire ${ }^{17}$.

La Reine mere se porte beaucoup mieux ${ }^{18}$. Son dernier accès n'a duré que deux petites heures; et l'appetit qu'elle avoit perdu luy est revenu. Vous aurés seû la maladie du Prince d'Espagne ${ }^{19}$ et celle de l'Empereur ${ }^{20}$. On acheva hier la Lecture de la production de M.r Talon ${ }^{21}$ contre Fouquet ${ }^{22}$. On lit présentement celle contre les Thresoriers de l'Espagne. On lira ensuite celle de M.r Fouquet, et toutes ces lectures dureront plus de deux mois. Ainsi le procés ne peut être terminé de plus de quatre. M.r de Montausier ${ }^{23}$ a ses provisions pour commander en Normandie, où il ira dans quinze jours. Vous sçavés le voyage de M.r de Beaufort ${ }^{24}$ à Toulon. L'affaire des Jansenistes, et des Jesuites est accommodée ${ }^{25}$. On a envoyé les articles de l'accommodement au Pape ${ }^{26}$. Je ne sçais point encore ce que portent ces articles. Je vous baise tres humblement les mains, et suis absolument à vous.

De Paris ce 22 Mars $1669^{27}$

J'ay reçu les deux Lettres qu'il vous a plû m'escrire au sujet du Pere Berthet ${ }^{28}$, dont je vous rends graces de toute mon affection; mais je n'ay point encore reçu la réponse de Monsieur le Cardinal Rospigliosi' ${ }^{29}$. Je l'attens avec d'autant plus d'impatience, que le tems qu'on avoit donné à ce Pere pour demeurer à Paris s'en va expirer. Je vous envoyerai par la premiere occasion mes origines de la Langue Italienne ${ }^{30}$. J'ay grande impatience d'en sçavoir vôtre jugement; et quand vous les aurés lües, je vous

(17) Il verso citato è tratto da Ad Guillelmum Lamonaeum, magistrum Libellorum supplicum (Cf. Lettres inédites à Pierre-Daniel Huet cit., pp. 187-188).

(18) Riguardo alla salute di Anna d'Austria, il 20 giugno Ménage aveva scritto: «La Reine Mere qui a toujours la fievre tierce, a pris aujourdhuy du vin emetique». (Lettres inédites à Pierre-Daniel Huet cit., p. 189).

(19) Carlo II d'Asburgo, erede al trono di Spagna, nato nel 1661 , era un bambino rachitico e malaticcio. Regnò dal 1665 al 1700.

(20) Leopoldo I d'Asburgo (1640-1705), eletto imperatore del Sacro Romano Impero nel 1658.

(21) Denis Talon (1628-1698), procuratore generale della corte di giustizia istituita contro Fouquet, si pronunciò per la condanna a morte, ma fu sostituito nel dicembre 1663 da Guy Chamillart.

(22) Il 9 giugno 1663 Fouquet era stato trasferito dal castello di Vincennes alla Bastiglia. Ménage gli rimase fedele anche negli anni della prigionia e, nel 1687 , rivolse al re una domanda di grazia in versi latini, De Fulketo ad Ludovicum Magnum (E. SAMFIRESCO, op. cit., p. 39).

(23) Charles de Saint-Maure, marchese di Montausier (1610-1690), divenne governatore della Normandia nel 1663. Due anni dopo fu creato duca e pari di Francia. Fu Montausier, com'è noto, a ideare la Guirlande de Julie, di cui compose quindici madrigali, dei sessantadue che la costituiscono.

(24) François de Bourbon-Vendôme, duca di Beaufort (1616-1669). Dopo aver partecipato alla Fronda, si distinse come ufficiale di marina nella lotta contro i pirati barbareschi. Cadde in com- battimento mentre portava soccorso ai veneziani, assediati dai turchi a Candia.

(25) Nei primi mesi del 1663 , ebbero luogo a $\mathrm{Pa}$ rigi, su iniziativa dell'arcivescovo Comminges e col beneplacito del re, alcuni incontri tra gesuiti e giansenisti, con lo scopo di trovare un accordo sui principali contrasti dottrinali. A queste conversazioni non prese però parte Arnauld, diffidente nei confronti dei gesuiti e contrario a qualunque soluzione di compromesso. In sua assenza, gli altri rappresentanti giansenisti si ridussero ad inviare a Roma un'esposizione delle proprie opinioni, rimettendosi esplicitamente al giudizio del papa. Quest'ultimo rispose con un 'breve' a tutti i vescovi di Francia, nel quale ribadiva le precedenti condanne delle tesi gianseniste. La trattativa si risolse quindi con un nulla di fatto e con una ripresa ancora più violenta delle polemiche. (Cf. SAInTe-Beuve, Port-Royal, éd. M. Leroy, Paris, Gallimard, Bibliothèque de la Pléiade, 1952-1955, Tome II, pp. 649-660).

(26) Alessandro VII, al secolo Fabio Chigi (1599-1667), eletto papa nel 1655, condannò il giansenismo nelle encicliche Ad sacram (1656) e Regiminis apostolici (1665).

(27) Epistolae cit., p. 257.

(28) Il padre Berthet, gesuita, aveva collaborato con Ménage alla stesura delle Origini della lingua italiana (E. SAMFIRESCO, op. cit., p. 132).

(29) Giacomo Rospigliosi, creato cardinale nel 1667, fu internunzio a Bruxelles e legato pontificio ad Avignone e Ferrara. Morì nel 1684.

(30) Le origini della lingua italiana, Paris, Sebastien Mabre-Cramoisy, 1699. 
prie de me le dire sincerement. J'en ay destiné un Exemplaire à M.r le Cardinal Rospigliosi; ne sçachant par qui le luy faire tenir, je le consigneray au premier jour entre les mains de M.r le Nonce. M.r de Valois estime fort vôtre Livre, et il doit vous êcrire au premier jour pour vous en dire son avis. Je vous demande toujours la continuation de vôtre amitié, et suis toujours absolument à vous.

Menage 